\title{
Temporal discrimination of visual stimuli in pigeons
}

\author{
HIROSHI YAMASHITA \\ Kyoto University, Kyoto, Japan
}

\begin{abstract}
Characteristics of the discrimination of brief visual stimuli were examined in three pigeons by a psychophysical procedure. In a two-choice discrimination task, the difference between the duration to be compared and the standard duration, which yielded $75 \%$ correct choice responses, was determined as the difference threshold for each of four standard durations ranging from .25 to $1.0 \mathrm{sec}$ by a variant of the up-down method. It was found that the difference threshold was roughly proportional to the standard duration: Weber's law provided a reasonable approximation. The value of the Weber fraction was about .25, which is in accord with the results of discrimination experiments with longer durations in pigeons.
\end{abstract}

Duration is a discriminable property of a stimulus. Duration discrimination is regarded as one of the important methods in the study of human time perception (Allan, 1979). Stubbs (1968) first used an operant choice technique in the study of duration discrimination in pigeons, which is comparable to a single-stimulus design in human experimentation. Many experiments have been performed with animals, and the operant choice method seems to be well established (Richelle \& Lejeune, 1980). In a typical experiment, after the animal makes an "observing" response and a duration stimulus (tone or light) is presented, two choice alternatives are given to the animal to make a choice, "long" or "short." According to the stimulus duration, correct responses are reinforced with food.

To determine whether there are common processes underlying human and animal performance, and whether a general theory is possible, it is necessary to obtain comparable data. An important difference between studies of human and animal temporal discrimination is that virtually all of the human experimentation concerns very short time intervals, generally in the millisecond range. Studies with animals, however, which stem from the study of performance under temporal schedules, such as FI (fixed interval) or DRL (differential reinforcement of low rate) schedules, typically use values of $10 \mathrm{sec}$ or longer.

In the present study, the characteristics of discrimination of brief time intervals in pigeons were examined. In a two-choice discrimination task, difference thresholds for durations shorter than $1.0 \mathrm{sec}$ were determined using a variant of the up-down method developed for use with rats by Church, Getty, and Lerner (1976).

I would like to thank R. Motoyoshi and T. Hirano of Kyoto University for their helpful advice during the course of the experiment, and $S$. Chase of Hunter College and E. Tobach of The American Museum of Natural History for their comments on the manuscript.

Requests for reprints should be sent to Hiroshi Yamashita, Department of Mammalogy, The American Museum of Natural History, Central Park West and 79th Street, New York, NY 10024.

\section{METHOD}

\section{Subjects}

Three male homing pigeons (Columba livia) were maintained at $80 \%$ of their free-feeding weights. All had had some previous experience with reinforcement schedules, and two of the pigeons (P141 and P142) had also previously served as subjects in an experiment similar to the present one (Yamashita, 1982).

\section{Apparatus}

A sound-attenuating experimental chamber for pigeons, with internal dimensions of $30 \times 35 \times 35 \mathrm{~cm}$, was used. Three response keys, $2.5 \mathrm{~cm}$ in diameter, were mounted behind openings in an aluminum panel $8.0 \mathrm{~cm}$ apart, center to center, $26 \mathrm{~cm}$ above the floor. The center key could be transilluminated by white light, the side keys by blue lights. A red-light-emitting diode, $0.3 \mathrm{~cm}$ in diameter, was mounted on the panel $1.0 \mathrm{~cm}$ above the center key. The duration of illumination of this lamp provided the discriminative stimuli to be used in these experiments. Grain could be presented by raising a food magazine mounted behind an aperture on the panel. The feeder was illuminated when food was available. A dim houselight illuminated the chamber throughout the experimental session except during reinforcement. The behavior of the subject could be observed through a small hole located in the wall opposite the working panel. A microprocesser controlled events in the experimental chamber and recorded the data.

\section{Procedure}

General procedure. A trial began with the center key illuminated and the other two dark. A single peck on the center key turned its light off and the signal lamp on. The signal lamp remained on for one of the two possible durations. When the signal lamp went off, the two side keys were illuminated simultaneously. A single peck on either key turned the keylights off. If the pigeon pecked the left key when the signal duration had been the shorter one or the right key when the signal duration had been the longer one, it was reinforced with food or a brief magazine light (conditioned reinforcer). After a 15-sec intertrial interval (ITI) the next trial began. No correction trial for an incorrect response was given. Responses during the ITI had no effect. Correct responses were reinforced with food according to a modified variable-ratio (VR) schedule (Stubbs, 1976). The pigeons had to respond correctly on a few trials before food was available (VR 1.7 schedule), and the specific choice response that produced food was predetermined and changed nonsystematically from reinforcement to reinforcement. 


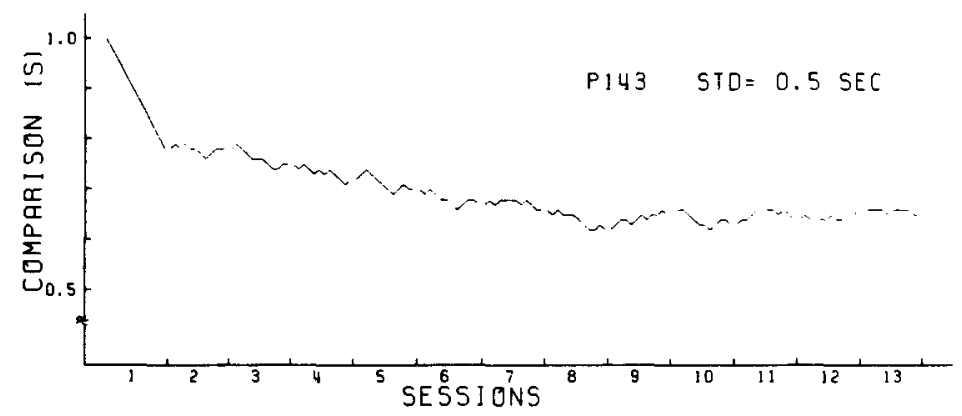

Figure 1. Duration of the comparison as a function of sessions for P143 with a 0.5 -sec standard.

Until the ratio was completed and the preselected choice response occurred, all correct-choice responses only produced the magazine light for $0.7 \mathrm{sec}$. Food reinforcement consisted of a $4.0-\mathrm{sec}$ presentation of the magazine.

Each session was divided into 12 16-trial blocks (192 trials). The two signal durations appeared equally often in a randomly permutated order in each block with the restriction that the same duration never appeared in more than three trials in a row.

Preliminary training. A fading procedure (Kinchla, 1970; Stubbs, 1968) was used to train the subjects. After chained responses, pecking the center key and then the lighted side key, were established, two discriminative stimuli $(0.5$ and $1.0 \mathrm{sec}$ in duration) were introduced. At first, only the "correct" side key was lit when the center key was pecked; a peck on the lighted side key was reinforced. The brightness of the "incorrect" side key was gradually increased in 12 steps over about 30 sessions, until it was equal to that of the "correct" side key. Partial reinforcement of the correct responses was introduced after duration discrimination was well established. A detailed description of the shaping procedure is given in Yamashita (1982).

Determination of the difference threshold. Threshold determination consisted of three phases: discrimination training, testing, and transition. Before obtaining difference thresholds for each standard, discrimination training was performed with the comparison duration twice as long as the standard (except for a 1.8-sec comparison for the 1.0-sec standard for P141 and P143 and for the redetermination for $P 142$ ). Training with each standard was at least five sessions in length. Training was continued until the percentage correct exceeded $90 \%$ on at least three consecutive sessions. In the testing phase, the shorter duration was held constant. This duration is referred to as the standard. The longer duration (comparison) was manipulated according to the performance of the subject. After each block of 16 trials, the duration of the comparison was redetermined. If $75 \%$ of the choice responses on the previous 16 trials (i.e., 12) were correct, the duration of the comparison stimulus was unchanged. If more than $75 \%$ of the responses were correct, the duration of the comparison was reduced by some constant (10 msec; larger values were sometimes used in the first few sessions of the testing). If fewer than $75 \%$ of the responses were correct, the duration of the comparison was increased by the same constant. In this way, the duration difference that could be correctly identified on $75 \%$ of the trials, the difference threshold, was determined.

In the first session of the testing, and at the start of each subsequent testing session, the comparison began at the value used in the last block of the preceding session. At least 10 sessions of testing were given with each standard. Testing ended when no new comparison value was used for 5 consecutive sessions. The data obtained from these 5 sessions (the determination period) were used for the difference thresholds reported here. Difference thresholds were obtained for standard durations of $0.25,0.5,0.75$, and $1.0 \mathrm{sec}$. The order in which these standards were used is shown in Table 1.
Several transition sessions were inserted between the testing and the next discrimination training with the new standard. If the new standard was shorter, the standard was set at the new value and the comparison was not changed in the first transition session but gradually decreased to the new value, twice as long as the standard, session by session. This procedure was used to minimize disruption of the performance. If the new standard was longer, the comparison was set at the new value, and the shorter duration was increased to the value of the new standard.

\section{RESULTS}

An example of the course of testing is shown for P143 with the 0.5 -sec standard in Figure 1. The duration of the comparison signal is plotted as a function of 16-trial blocks. The comparison could be decreased rapidly at first since accuracy remained above $75 \%$. Further decrease in the duration of the comparison required more extensive training to maintain accuracy at $75 \%$.

The results of testing are summarized in Table 1. From the five sessions of the determination period (i.e., 55 blocks, excluding the first block of each session), the median of the difference between the comparison and the standard durations $(\Delta T)$ was calculated for each standard

Table 1

Order of Testing, Minimum (Min) and Median (Med) Difference between the Comparison and the Standard Durations $(\Delta T)$, and Range of Difference at Each Standard $(T)$

\begin{tabular}{llcccc}
\hline & & & \multicolumn{2}{c}{$\Delta T^{*}$} & \\
\cline { 4 - 6 } Animal & $T^{*}$ & Order & Min & Med & Range \\
\hline P141 & .25 & 4 & .06 & .09 & 5 \\
& .5 & 3 & .06 & .10 & 9 \\
& .75 & 1 & .16 & .18 & 7 \\
& .75 & 5 & .15 & .17 & 4 \\
& 1.0 & 2 & .27 & .29 & 4 \\
P142 & 1.0 & 6 & .22 & .26 & 8 \\
& .25 & 2 & .05 & .08 & 5 \\
& .5 & 3 & .12 & .14 & 6 \\
& .75 & 4 & .15 & .17 & 3 \\
& 1.0 & 1 & .34 & .38 & 6 \\
P143 & 1.0 & 5 & .17 & .26 & 15 \\
& .25 & 3 & .05 & .09 & 6 \\
& .5 & 2 & .12 & .15 & 4 \\
& .5 & 5 & .12 & .15 & 5 \\
& .75 & 4 & .11 & .13 & 4 \\
& 1.0 & 1 & .21 & .29 & 9 \\
\hline
\end{tabular}

Note-Data were taken from the last five sessions at each standard. The range represents the number of $10-\mathrm{msec}$ steps. $\quad$ *In seconds. 

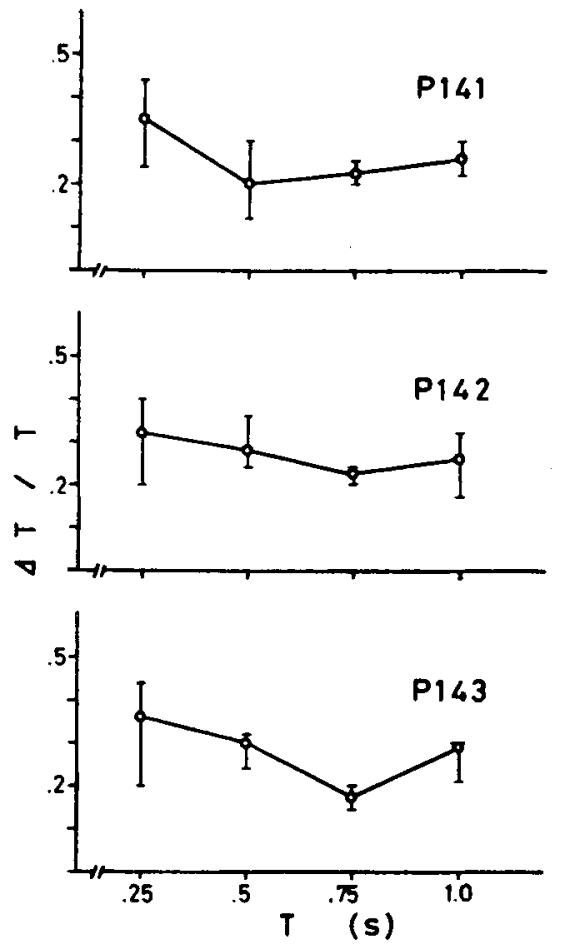

Figure 2. Weber fraction $(\Delta T / T)$ as a function of the standard duration $(T)$ for each subject. The vertical lines indicate the ranges corresponding to those shown in Table 1.

$(T)$. The minimum difference, for which the subject made at least $75 \%$ correct responses in a session, is also shown even if this occurred before the determination period. In order to show the variability of the performance, the range of the comparison value that appeared during the determination period is shown in terms of the number of 10msec steps. The difference threshold increases as the standard duration increases for each subject, except for one case (P143, between the 0.5- and 0.75-sec standards). Similar values were obtained from the 3 subjects.

The Weber fraction, $\Delta T / T$, is shown in Figure 2 as a function of the standard duration. The vertical lines indicate the range shown in Table 1. For the standard durations tested twice, the smaller value is shown. It is assumed that the method of determining the difference threshold eliminates small values obtained only by chance and that distracting factors will work in the direction of increasing the value of the difference threshold. The value of the Weber fraction may be regarded as being relatively constant, when the variability is taken into consideration. The relatively large variability of the Weber fraction for short standard durations may be attributable to the fact that the step size was constant $(10-\mathrm{msec})$ for all the standard durations. The variability is smallest with the $0.75-$ sec standard, and it seems possible that the curves have a minimum between 0.5 and $0.75 \mathrm{sec}$, but, because of the small number of points, a definite statement cannot be made.
A linear function, $\Delta T=k T+s$, was fitted to the difference threshold (median $\Delta T$ ) data by the method of least squares criterion. $T$ and $\Delta T$ stand for the standard duration and the corresponding difference threshold. The smaller $\Delta T$ was taken when two values were available. The slope, $k$, and the intercept, $s$, of this function were .232 and .01 for P141, .228 and .02 for P142, and .232 and .02 for P143. The variance accounted for was $90.9 \%$, $96.3 \%$, and $74.1 \%$, respectively, for the three subjects. It remained virtually unchanged $(90.6 \%, 94.9 \%$, and $72.9 \%$, respectively) when the best-fitting line with zero intercept was recalculated, $\Delta T=k T$. This means that the results are compatible with the simple version of Weber's law.

Behavioral observations showed that all subjects often pecked the dark center key during the ITI, but that they stopped pecking when the signal lamp was illuminated by the first peck after the illumination of the center key, and that they rarely pecked it during the signal presentation. No stereotyped behavior during the signal, which might mediate the choice response, was observed. Choice responses were made without delay.

\section{DISCUSSION}

It was demonstrated that the pigeons were able to discriminate among relatively short durations of visual stimuli. Discriminability in terms of the Weber fraction is roughly constant for the durations ranging between 0.25 and $1.0 \mathrm{sec}$.

The value of the Weber fraction obtained in this experiment $(0.25)$ is in accord with the values obtained with longer durations in discrimination experiments (e.g., Perikel, Richelle, \& Maurissen, 1974; Stubbs, 1968) and in temporal differentiation schedules (e.g., Catania, 1970) in pigeons. Further research is needed to see if the same process functions in the discrimination of durations of different ranges and if the value around 0.25 represents something specific to temporal discrimination in pigeons.

Church et al. (1976) determined difference thresholds in rats for durations ranging from 0.5 to $8.0 \mathrm{sec}$. They found that the Weber fraction increased below $1.0 \mathrm{sec}$. This might be attributable to a species difference between rats and pigeons, but slight differences in the procedure should also be considered. Although the same technique was used to determine the difference threshold, one important procedural difference is that the stimulus was initiated by the subject in the present experiment but not in Church et al.'s. When the subject itself produces the stimulus, the duration of exposure to a stimulus is probably less variable. If this source of variability is independent of the duration of the standard, its effects should be more pronounced for shorter durations. Church et al., taking this into account, described their results by using Getty's (1975) generalized Weber model, which incorporated the nontemporal factor as a constant component of variance, and argued that the same model could be ap- 
plied to data from human subjects and rats. But it may be necessary to obtain data by minimizing the variance associated with extraneous factors before developing a formal model.

The conclusion that Weber's law holds for the range of durations studied here must be tentative since the small number of data points does not lend itself to the evaluation of different models. Deviation from proportionality may be expected for shorter durations. More data is needed before a more definite statement can be made. In order to explore the characteristics of the discrimination function for much shorter durations, a further refinement of the experimental technique may be required.

It has been argued that Weber's law does not necessarily hold in human duration discrimination. It appears to hold only for durations between 0.2 and $2.0 \mathrm{sec}$ (Getty, 1975). The range of duration examined in this study, however, falls in the range in which the Weber fraction is relatively constant in human data. Similarity of the discrimination function for the same range of durations, indicated in the results of this study, suggests that the pigeons respond to the temporal aspect of the stimuli in a way that is somewhat similar to that of humans. The existence of the same kind of process, then, may be inferred. Getty (1975) obtained the value of 0.05 as the Weber fraction with human subjects. The difference in the value of the Weber fraction can be partly attributable to the difference in method. The single-stimulus design employed in this study may be considered to be a more difficult task than the forced-choice design often used in human experiments. Although it was shown that with an appropriate method pigeons could be trained to make rather difficult discriminations, much more extensive experimentation is necessary for a more detailed analysis and comparison among species.

\section{REFERENCES}

Allan, L. G. (1979). The perception of time. Perception \& Psychophysics, 26, 340-354.

Catania, A. C. (1970). Reinforcement schedules and psychophysical judgments: A study of some temporal properties of behavior. In W. N. Schoenfeld (Ed.), The theory of reinforcement schedules (pp. 1-42). New York: Appleton-Century-Crofts.

Church, R. M., Getty, D. J., \& Lerner, N. D. (1976). Duration discrimination by rats. Joumal of Experimental Psychology: Animal Behavior Processes, 2, 303-312.

GETTY, D. J. (1975). Discrimination of short temporal intervals: A comparison of two models. Perception \& Psychophysics, 18, 1-8.

KINCHLA, J. (1970). Discrimination of two auditory durations by pigeons. Perception \& Psychophysics, 8, 299-307.

Perikel, J. J., Richelle, M., \& Maurissen, J. (1974). Control of key pecking by stimulus duration. Journal of the Experimental Analysis of Behavior, 22, 131-134.

RICHELLE, M., \& LEJEUNE, H. (1980). Time in animal behavior. New York: Pergamon Press.

STUBBs, D. A. (1968). The discrimination of stimulus durations by pigeons. Journal of the Experimental Analysis of Behavior, 11, 223-238.

STUBBs, D. A. (1976). Response bias and discrimination of stimulus duration. Joumal of the Experimental Analysis of Behavior, 25, 243-250.

Yamashita, H. (1982). Discrimination of brief visual durations in pigeons (Brief Reports No. 19). Kyoto, Japan: Kyoto University.

(Manuscript received February 10, 1986; revision accepted for publication June 4, 1986.) 